\title{
Four-Dimensional Computed Tomography Scanning for Dynamic Wrist Disorders: Prospective Analysis and Recommendations for Clinical Utility
}

\author{
${ }^{1}$ College of Medicine and Public Health, Flinders University, Adelaide, \\ Australia \\ 2 Department of Orthopaedic and Upper Limb Surgery, Tauranga \\ Hospital, Tauranga, New Zealand \\ ${ }^{3}$ Department of Medical Imaging, Repatriation General Hospital, Daw \\ Park, South Australia, Australia \\ ${ }^{4}$ Division of Hand and Upper Limb Surgery, Department of \\ Orthopaedic Surgery, Flinders University, Adelaide, Australia
}

Renee Carr, BSc ${ }^{1}$ Simon MacLean, MBChB, FRCS (Tr\&Orth), PGDipCE ${ }^{2}$

John Slavotinek, MBBS, BSc, FRANZCR ${ }^{3}$ Gregory I. Bain, PhD, MBBS, FRACS, FA (Ortho)A ${ }^{4}$

\author{
Address for correspondence Gregory I. Bain, PhD, MBBS, FRACS, FA \\ (Ortho)A, Department of Orthopaedic Surgery, Flinders University, \\ Bedford Park, South Australia 5042, Australia \\ (e-mail: greg@gregbain.com.au).
}

J Wrist Surg 2019;8:161-167.

\begin{abstract}
Keywords

- 4D CT

- four-dimensional computed tomography

- dynamic CT

- dynamic wrist disorders

- dynamic instability disorders

Background Four-dimensional computed tomography (4D CT) is a rapidly developing diagnostic tool in the assessment of dynamic upper limb disorders. Functional wrist anatomy is incompletely understood, and traditional imaging methods are often insufficient in the diagnosis of dynamic disorders.

Technique This study has developed a protocol for 4D CT of the wrist, with the aim of reviewing the clinical utility of this technology in surgical assessment. A Toshiba Aquilion One Vision scanner was used in the protocol, in which two- and threedimensional "static" images, as well as 4D "dynamic" images were produced and assessed in the clinical context of each patient. These consisted of a series of multiple 7second movement clips exploring the nature and range of joint motion.

Patients and Methods Nineteen patients with symptoms of dynamic instability were included in the study. Patients were assessed clinically by two orthopaedic surgeons, and qualitative data were obtained from radiological interpretation.

Results The study demonstrated varied abnormalities of joint movement attributed to a range of wrist pathology, including degenerative arthritis, ligamentous injuries, Kienbock's disease, and pain following previous surgical reconstructive procedures. Interpretation of the $4 \mathrm{D}$ CT scan changed the clinical diagnosis in 13 cases (68.4\%), including the primary $(15.8 \%)$ or secondary diagnosis $(52.6 \%)$. In all cases, the assessment of the dynamic wrist motion assisted in understanding the clinical problem and led to a change in management in 11 cases (57.9\%). The mean effective radiation dose for the scan was calculated at $0.26 \mathrm{mSv}$. Conclusion We have found that the clinical utility of 4D CT lies in its ability to provide detailed information about dynamic joint pathology not seen in traditional imaging, targeting surgical treatment. Limitations to the use of 4D CT scan include lack of availability of the technology, potential radiation dose, and radiographer training requirements, as well as limited understanding of the nature of normal motion.
\end{abstract}

received

May 20, 2018

accepted

September 24, 2018

published online

November 14, 2018
Copyright $\odot 2019$ by Thieme Medical Publishers, Inc., 333 Seventh Avenue, New York, NY 10001, USA. Tel: +1(212) 584-4662.
DOI https://doi.org/

10.1055/s-0038-1675564. ISSN 2163-3916. 
The diagnosis of dynamic wrist disorders currently relies largely on clinical findings, as patients with dynamic instability often have normal appearances on conventional imaging studies, including plain radiographs, magnetic resonance imaging (MRI), and ultrasound. ${ }^{1,2}$ The current gold standard for diagnosis of intra-articular pathology is joint arthroscopy, a relatively invasive and expensive procedure, and it has been suggested that four-dimensional computed tomography (4D CT) can provide an alternative method of assessment of patients with suspected instability. 2,3

The previous methods of assessing dynamic disorders of the wrist have included plain radiographs and fluoroscopy. ${ }^{4,5}$ Examples of radiographs include those in radial and ulnar deviation, and clenched fist views. ${ }^{6}$ Fluoroscopy can be used to assess carpal motion and instability, ${ }^{6}$ aid in intra-articular injections, ${ }^{7}$ and be of value in the operating theater. ${ }^{5}$

Furthermore, while conventional static imaging methods can provide information about carpal bone anatomy and alignment, dynamic imaging has the advantage of assessing the carpus, distal radius and ulna, and joint spaces throughout the range of movement. ${ }^{8}$

Accurate diagnosis of dynamic disorders such as carpal bone instability is essential; early diagnosis of dynamic instability and appropriate treatment can prevent progression to static instability and osteoarthritis. ${ }^{9}$ Moreover, if surgical intervention is deemed appropriate, the source of the instability must be appropriately identified. Detailed knowledge of the nature of instability will allow clinicians to offer more specific and appropriate surgery for each patient.

4D CT is an emerging diagnostic tool in the assessment of joint biomechanics, specifically dynamic upper limb disorders. Recent advances in CT scanning technology have provided the capacity to obtain three-dimensional (3D) images over time, the fourth dimension, and volume data obtained throughout the movement series can be reconstructed in two-dimensional (2D), 3D, and " $4 \mathrm{D}$ " formats, producing static images as well as dynamic cine-movies. $4 \mathrm{D}$ CT has previously been used in other disciplines, including perioperative planning for parathyroid and esophageal lesions. In the setting of orthopaedics, providing information about bone position throughout the range of joint movement has great implications for surgical planning and diagnostics. Research to date has demonstrated the feasibility of 4D CT for use in imaging upper limb joints and for the diagnosis and surgical planning of costoclavicular impingement, scapholunate instability, capitate subluxation, pisotriquetral instability, acromioclavicular dislocation, and snapping scapula. ${ }^{10-14}$ The technique has also been shown to produce images with high spatial and temporal resolution. ${ }^{15,16}$

\section{Technique}

The scanner used was a Toshiba Aquilion One Vision “320 slice” scanner (Toshiba, Tochigi, Japan). The table was centered to a position of $+70 \mathrm{~mm}$, with the volume range set to $140 \mathrm{~mm}$ to ensure that no table movement is required. The wrist was imaged $70 \mathrm{~mm}$ proximally and distally to the distal radioulnar joint (DRUJ). Slice thickness and interval were set to $0.5 \mathrm{~mm}$.
The scanning protocol included six dynamic 4D CT scans, one for each movement. The movement series consisted of flexionextension, radial-ulnar deviation, a clenched fist motion, a dartthrowing motion, reverse dart-throwing motion, and pronation-supination. Settings were $80 \mathrm{kV}$ tube voltage, $0.275 \mathrm{sec}-$ onds gantry rotation time, $50 \mathrm{~mA}$ current, and continuous X-ray. The total scan time for each movement was set to a default of 7.425 seconds to reduce radiation exposure. Radiographer monitoring the patient movements allowed the scan to be stopped and started on demand as each movement was completed. Data were reconstructed for each dynamic scan at 0.5second intervals, which resulted in 6 to 14 data volumes per movement.

The patient was positioned standing to the side of the scanner table, with the DRUJ centered in the scan volume. Their forearm was supported by a rubber rectangular block and was secured by Velcro straps attached to the table to reduce forearm movement and therefore minimize motion artifact. The investigator was positioned on the other side behind the scanner and passively guided the patient through the movement series, first on the asymptomatic wrist, used as a control, and then on the symptomatic wrist.

Both patient and investigator wore lead aprons, thyroid protectors, and lead glasses, and the investigator wore lead gloves when mobilizing the arm ( $\mathbf{F i g . 1}$ ).

Volume data were reconstructed using Toshiba software with $0.5-\mathrm{mm}$ thickness and $0.5-\mathrm{mm}$ interval between images, using both bone and soft tissue algorithms. Multiplanar reformations were produced using the bone algorithm in coronal, sagittal, and axial planes, and soft tissue reconstructions were used to produce the $4 \mathrm{D}$ images (i.e., 3D "movies"). A musculoskeletal radiologist reviewed the images and provided a radiological report.

\section{Patients and Methods}

Following ethics committee approval, 19 patients were invited to participate in the study and consented to a $4 \mathrm{D}$ CT scan. In preparation of the study, other senior surgical colleagues had raised concerns about proceeding with a surgical plan based on $4 \mathrm{D}$ scans, as we had not identified what was the normal motion. As such, the ethics committee agreed to allow the scans to be performed bilaterally.

These patients were selected on clinical grounds, such as symptoms suggestive of dynamic instability, impingement, or pain with motion of the wrist. Patient details including age, sex, and clinical diagnosis were recorded prior to scanning. The findings from 4D CT were assessment by a senior orthopaedic surgeon and musculoskeletal radiologist and then compared with the initial imaging and clinical diagnosis.

The effective dose of radiation was calculated using the method described by Biswas et al ${ }^{17}$ using data obtained from the patients enrolled in the study.

\section{Results}

Nineteen patients were enrolled in the study and underwent a bilateral $4 \mathrm{D} \mathrm{CT}$ of the wrist. The clinical indications and 

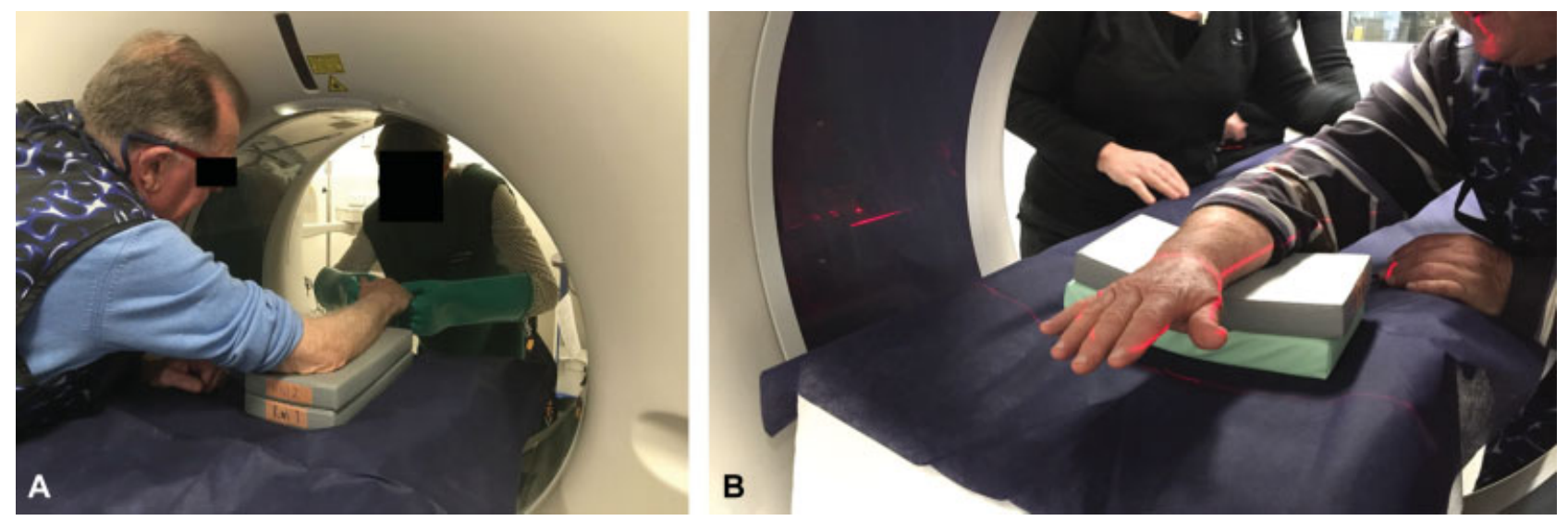

Fig. 1 Images demonstrating patient positioning in the scanner for a four-dimensional computed tomography wrist, with forearm stabilized on a rubber block and the investigator passively moving joint (A). The scanning plane is centered across the distal radioulnar joint (B).

pathologies identified as a result of 4D CT included osteoarthritis, instability, Kienbock's disease, ulnar impaction, and ulnar translocation. - Table 1 identifies the cases in which the $4 \mathrm{D}$ scan was used and how it affects the diagnosis, interpretation, and management of the wrist.

The assessment of the $4 \mathrm{D}$ CT scan leads to a change in the diagnosis in 13 cases (68.4\%), including a change in the primary diagnosis in 3 cases (15.8\%) and secondary diagnosis in 10 cases (52.6\%). In all cases, the 4D scan assisted in obtaining a better understanding of the clinical problem. The interpretation of the $4 \mathrm{D}$ scan leads to a change in management in $11(57.9 \%)$ cases.

The mean effective radiation dose was $0.26 \mathrm{mSv}$. The acquisition of appropriately orientated images is important. For example, radioulnar deviation is best imaged from anterior and/or posterior aspects to assess the DRUJ from the

Table 1 Clinical findings on 4D CT of the wrist

\begin{tabular}{|c|c|c|c|c|c|c|}
\hline \multicolumn{2}{|c|}{ Wrist } & \multicolumn{2}{|l|}{ Diagnosis } & \multicolumn{3}{|c|}{ Value of $4 \mathrm{D}$ CT scan } \\
\hline Case & $\begin{array}{l}\text { Patient's } \\
\text { age, gender }\end{array}$ & Before 4D CT scan & After 4D CT scan & Diagnosis & Interpretation & Management \\
\hline 1 & $57, \mathrm{~F}$ & LTAC & LTAC with minimal MC motion & - & + & + \\
\hline 2 & $46, \mathrm{~F}$ & Pain posttrapeziectomy, cause & Scaphotrapezoid OA and impingement & + & + & + \\
\hline 3 & $59, M$ & Pain post TFC repair, cause & DRUJ impingement but no instability & + & + & + \\
\hline 4 & $77, \mathrm{~F}$ & Ulnar pain and instability, STT OA & STT OA, SLI & + & + & + \\
\hline 5 & $74, F$ & Kienbock's, MC OA & Kienbock's disease and SLI & + & + & - \\
\hline 6 & $54, \mathrm{~F}$ & SLI & SLAC grade 1 & + & + & + \\
\hline 7 & $26, \mathrm{M}$ & Kienbock's disease & Proximal lunate collapse, coronal fracture. & - & + & - \\
\hline 8 & $53, \mathrm{~F}$ & Kienbock's disease & Normal motion wrist & + & + & + \\
\hline 9 & $73, F$ & DRUJ OA, instability & DRUJ OA, but no instability & + & + & - \\
\hline 10 & $49, M$ & DRUJ instability & Dorsal ulnar subluxation, $\mathrm{OA}$ & + & + & + \\
\hline 11 & $56, F$ & STT OA, FCR cyst & Trapezium impinges on distal scaphoid & - & + & - \\
\hline 12 & $33, M$ & Kienbock's disease & $\begin{array}{l}\text { Kienbock's disease, ulnotriquetral } \\
\text { and radioscaphoid impingement }\end{array}$ & + & + & + \\
\hline 13 & $79, M$ & DRUJ OA and instability & $\begin{array}{l}\text { Ulnocarpal impaction, } \\
\text { ulnar translocation, SLAC }\end{array}$ & ++ & + & + \\
\hline 14 & $79, \mathrm{M}$ & Pain postulnar hemiresection & Radioulnar abutment in full supination & - & + & - \\
\hline 15 & 49, M & SL or LT instability & Normal motion wrist & + & + & + \\
\hline 16 & $68, M$ & $\begin{array}{l}\text { Ulnar wrist pain, } \\
\text { Kienbock's disease }\end{array}$ & Kienbock's, ulnocarpal impaction & - & + & - \\
\hline 17 & $51, M$ & Kienbock's disease & Sclerotic, cystic lunate & - & + & - \\
\hline 18 & $19, M$ & Normal X-ray, SL instability & Gross SL instability & ++ & + & + \\
\hline 19 & $41, F$ & Pain, previous scaphoid fractures & $\begin{array}{l}\text { Mild SL widening, cyst and } \\
\text { subtle lunate fracture }\end{array}$ & ++ & + & + \\
\hline
\end{tabular}

Abbreviations: 4D CT, four-dimensional computed tomography; DRUJ, distal radioulnar joint; LT, lunotriquetral; LTAC, lunotriquetral advanced collapse; MC, midcarpal; OA, osteoarthritis; SLAC, scapholunate advanced collapse; SLI, scapholunate instability; STT, scaphotrapeziotrapezoidal; TFC, triangular fibrocartilage complex.

Note: changes in the primary $(++)$ and secondary $(+)$ diagnosis. 
proximal aspect, as seen from the interosseous membrane. The dart-throwing motion is best observed from an oblique angle, dorsal ulnar aspect, to ensure that the carpus is visualized from the correct orientation.

Specific processing techniques such as cropping the 4D image assisted in defining the details of the anatomy, particularly in assessing the DRUJ, where a direct view into the joint from the proximal aspect could be obtained.

\section{Discussion}

Wrist anatomy, function, and pathology have been incompletely understood, and many disorders have been reliant on clinical history and examination. The 4D CT scan assists in obtaining a better understanding of the clinical problem, with detailed information from the multiple static and dynamic images about the relative joint position throughout the range of motion. This provides a new insight into normal motion, joint stability, instability, impingement, and arthritis. This includes changing the primary and secondary diagnoses. But more importantly, the better understanding of the patient's wrist functional motion leads to a change in management of the patient.

We have seen examples of the 4D scan unmasking the abnormal joint motion and instability during physiological motion and loading with a clenched fist. Examples include joint impingement, instability with subluxation, and ulnar translocation. Osseous impingement was observed with motion of degenerate joints (e.g., scaphotrapeziotrapezoidal, radiocarpal, and distal radioulnar joint), and impaction of the distal ulna on the carpus and distal radius was also demonstrated. An abnormal locked position of the scaphoid was also identified in a patient with instability (-Fig. 2). In the patient who has persistent pain following surgery, the dynamic scan can be valuable. Examples include patients with instability or impingement following previous triangular fibrocartilage complex repairs, ulnar hemiresection, trapeziectomy, and limited wrist fusion.

The dynamic scan also assists in further defining some pathologies that are not well understood. An example is lunotriquetral advanced collapse, where the lunotriquetral interval was seen to open during radial deviation (-Fig. 3 , - Video 1). Also, in Kienbock's disease, we have observed the impaction fracture of the lunate and how it corresponds with shape of the radius template, especially with a clenched fist (-Fig. 4, -Video 2).

\section{Video 1}

A 57-year-old female with suspected lunotriquetral advanced collapse. Dynamic two-dimensional computed tomography images demonstrate midcarpal osteoarthritic change, bony erosion of the capitate (A), and abutment of dorsal lunate osteophytes on the capitate in extension (B). Online content including video sequences viewable at: www.thiemeconnect.de/ products/ejournals/html/10.1055/s-0038-1675564.

\section{Video 2}

A 21-year-old male with Kienbock's disease. Fourdimensional image demonstrating a "radial template" forming on the proximal surface of the lunate $(\mathbf{A})$, as well as loss of medullary trabeculae highlighted in a radioulnar deviation view (B) and fragmentation and subchondral cyst formation $(\mathbf{C})$. Online content including video sequences viewable at: www.thiemeconnect.de/products/ejournals/html/10.1055/s0038-1675564.

Having reviewed the clinical use of the 4D CT scan, we are now able to make a comparison with fluoroscopy. Fluoroscopy provides a low-resolution 2D image of the full thickness of the wrist, as it is mobilized. The 4D CT scanning provides a dynamic 3D image of the wrist, which allows an
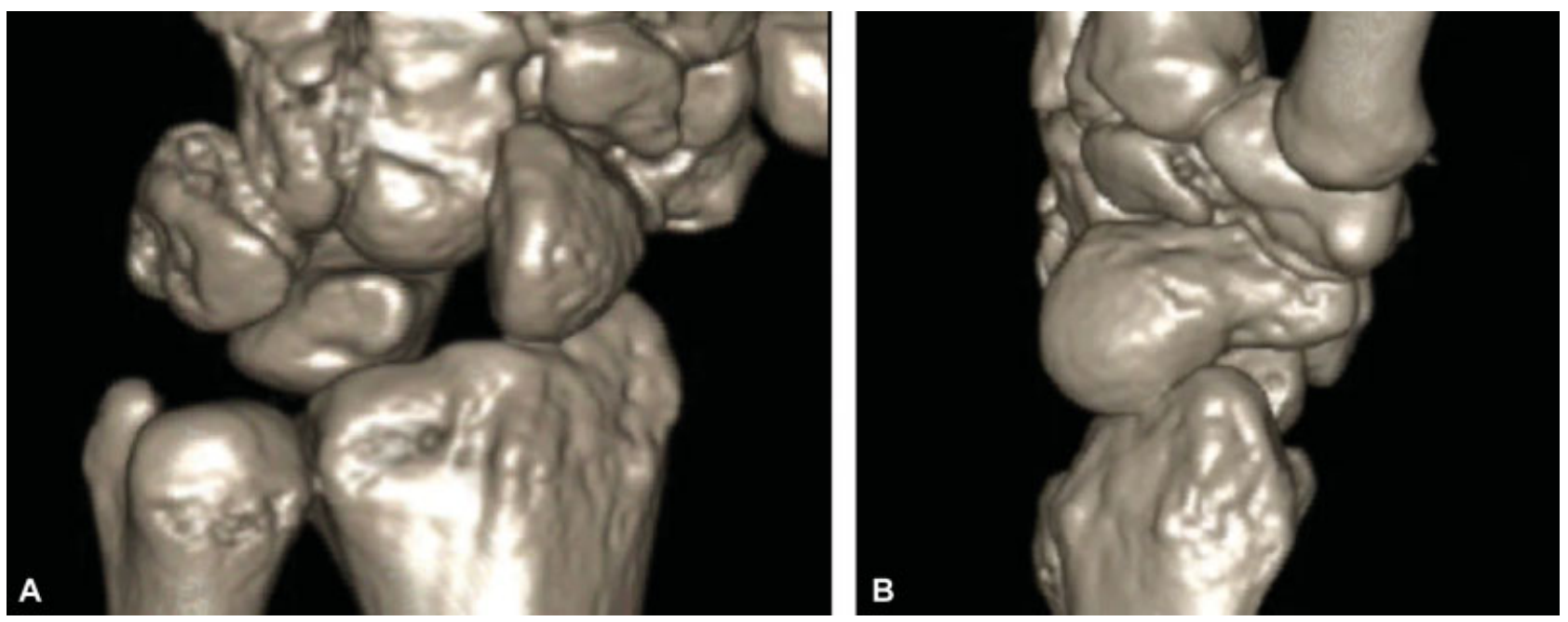

Fig. 2 A 19-year-old male presented with stiffness in flexion-extension following wrist trauma. Four-dimensional computed tomography demonstrates widening of the scapholunate interval (A) as well as an abnormal locked position of the scaphoid (B). 

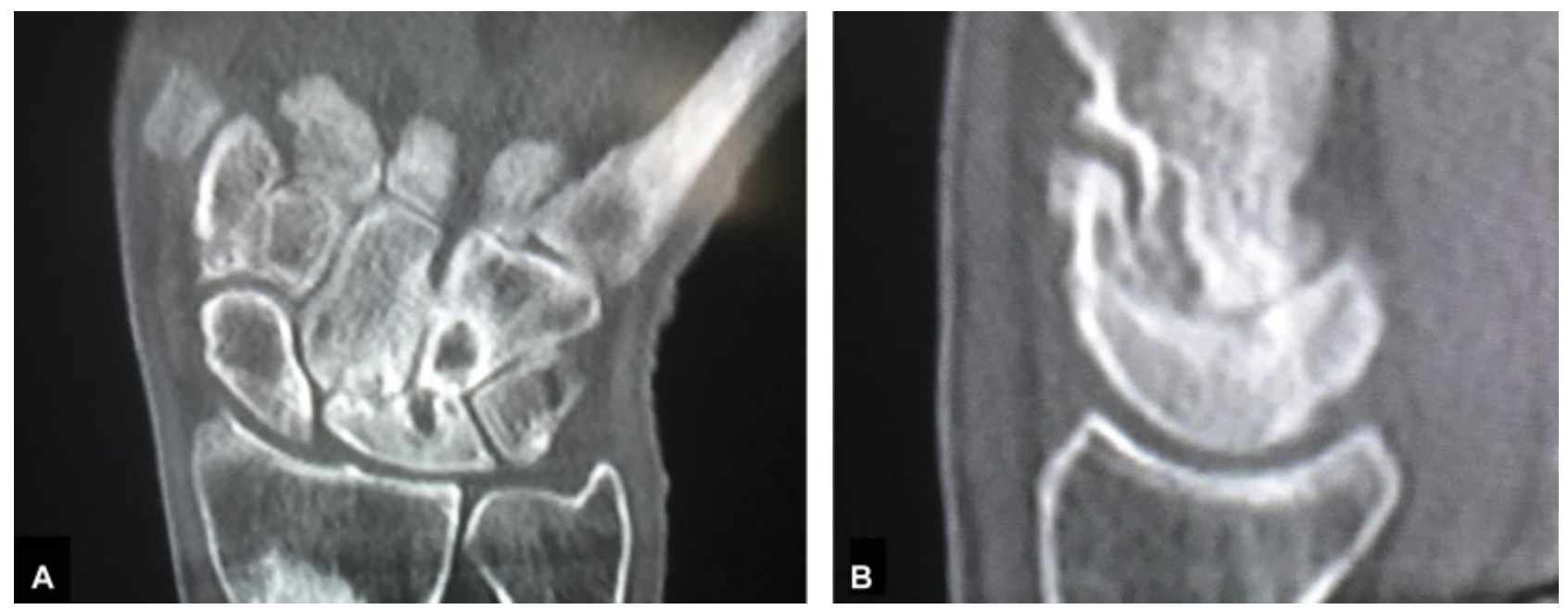

Fig. 3 A 57-year-old female with suspected lunotriquetral advanced collapse. Dynamic two-dimensional computed tomography images demonstrate midcarpal osteoarthritic change, bony erosion of the capitate (A), and abutment of dorsal lunate osteophytes on the capitate in extension (B).
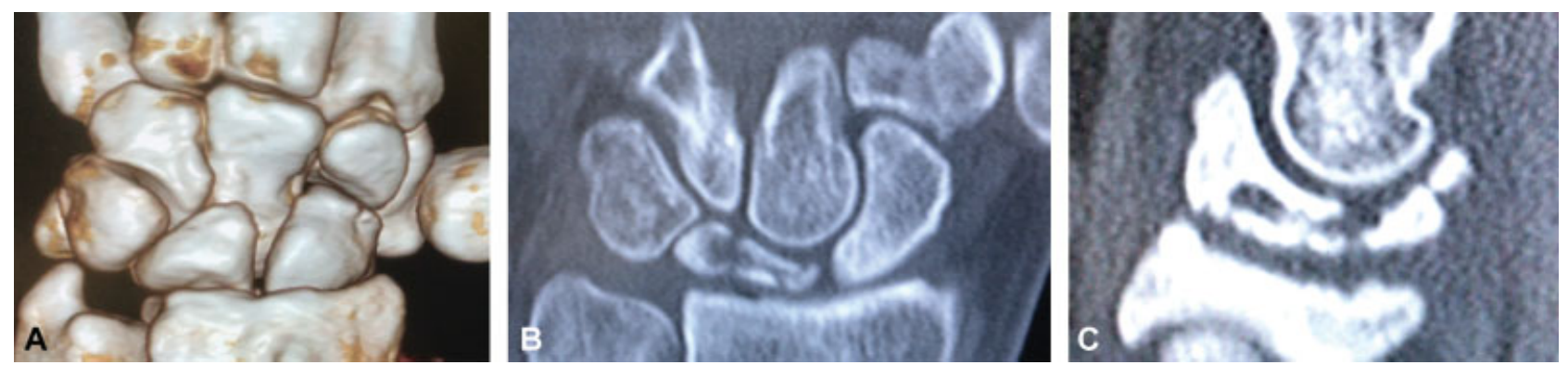

Fig. 4 A 21-year-old male with Kienbock's disease. Four-dimensional image demonstrating a "radial template" forming on the proximal surface of the lunate (A), as well as loss of medullary trabeculae highlighted in a radioulnar deviation view (B) and fragmentation and subchondral cyst formation (C).

overriding appreciation of the carpal motion. The dynamic 2D image is similar to conventional fluoroscopy but with a few important exceptions. Once the scan has been performed, the DICOM (Digital Imaging and Communications in Medicine) data can be manipulated and reformatted in any plane to provide the ideal 3D or 2D dynamic images. In addition, the thickness of the zone of the fluoroscopic assessment can be changed, depending upon the pathology to be assessed. For example, the fluoroscopic image can be focused on the area of interest. An example includes the "thin" coronal dynamic images of the scapholunate interval with the dynamic clenched fist view. Furthermore, dynamic 2D images only include a narrow window of the carpus and therefore provide a more localized assessment. This was seen to be of value when assessing dynamic scapholunate instability with the clenched fist or radioulnar deviation. It clearly demonstrated that in Kienbock's disease, the proximal and distal subchondral bone plates move separately through the site of the fracture.

Provocation testing in 4D CT clearly highlights its utility; the patient could demonstrate instability during the scan by performing basic maneuvers such as clenched fist (-Video 3 ), flexion-extension, or dynamic tests such as Watson's scapho- lunate instability test. This was useful in instability of the scapholunate interval, midcarpal joint, and DRUJ.

\section{Video 3}

Dynamic computed tomography (CT) in clenched fist motion demonstrating instability. Online content including video sequences viewable at: www. thiemeconnect.de/products/ejournals/html/10.1055/ s-0038-1675564.

Challenges to the implementation of 4D CT scanning in our hospital were varied. When performing wrist maneuverers, patient education and compliance are required to ensure that the patient moves the wrist correctly, within the 16-cm gantry, when the scanner is activated. If patient movement was too quick, this produced motion artifact and movements that were too slow and may exceed the scanner acquisition time with the loss of images during the latter part of the motion. Performing provocation maneuvers in the scanner can be difficult due to 
Table 2 Recommendations for 4D CT scan of the wrist

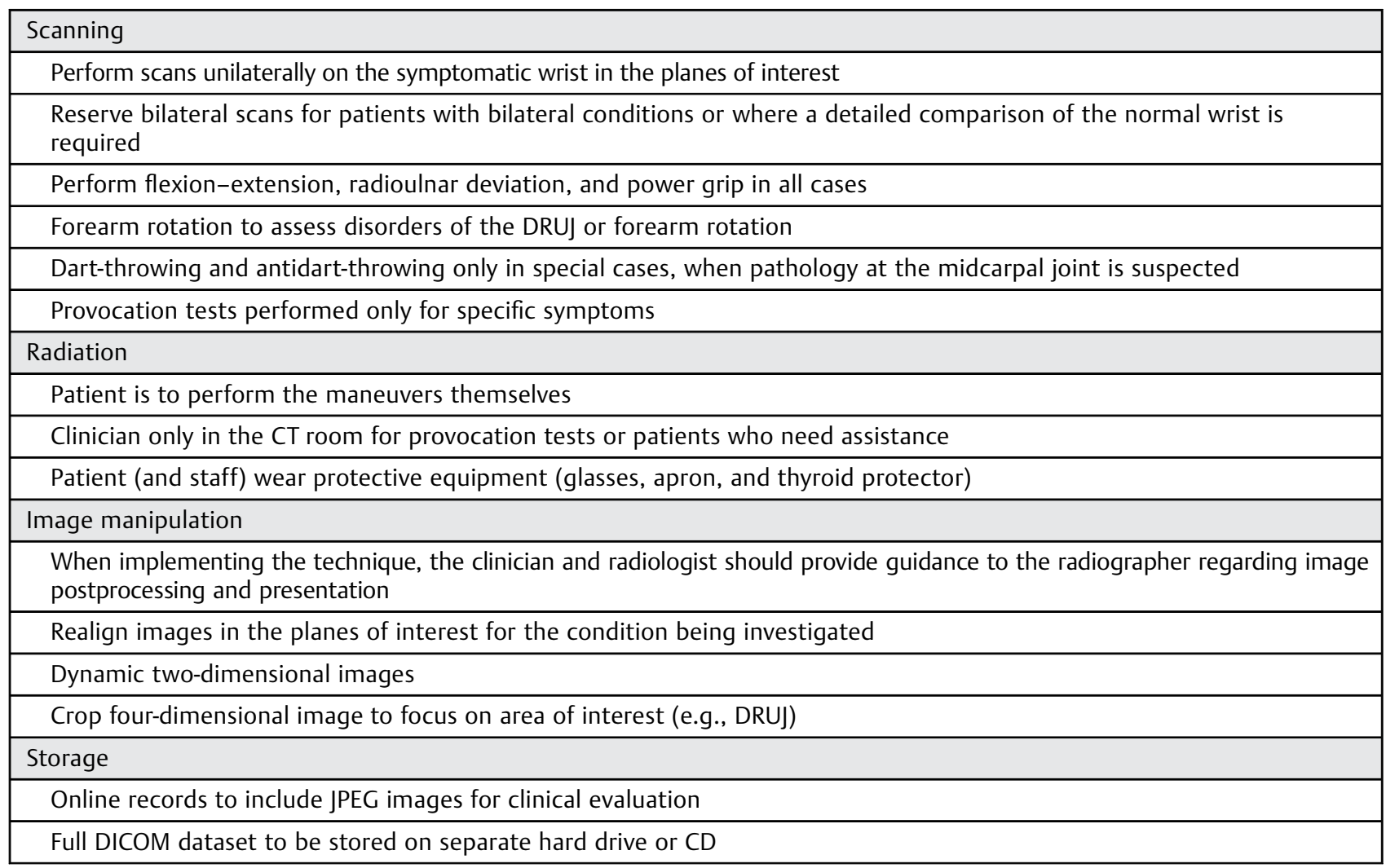

Abbreviation: CT, computed tomography; 4D CT, four-dimensional computed tomography; DICOM, Digital Imaging and Communications in Medicine; DRUJ, distal radioulnar joint.

patient discomfort and the confined space. Training of the radiography staff is important to produce quality $\mathrm{CT}$ images and to obtain operational efficiency in a busy hospital. Initially, the scans were delayed due to patient setup and education, donning radiation protection equipment and coordination of scanning with staff.

Image acquisition must be followed by correct processing and presentation, relevant to the condition being investigated. Data storage is a particular challenge, as the large data volume takes an extended time to process and requires considerable space on storage drives. Furthermore, access to experienced musculoskeletal radiologists is important, as they too are still learning this new imaging modality. A summary of our logistic recommendations is provided in - Table 2.

In conclusion, 4D CT is proposed as a useful adjunct in clinical evaluation of dynamic upper limb disorders, including instability and impingement, and can provide important insight regarding functional anatomy and its impact on disease progression.

\section{Conflict of Interest}

None declared.

\section{Acknowledgments}

We thank the radiography staff at Repat Radiology, SA Medical Imaging, Repatriation General Hospital, Daw Park, South Australia, Australia, for their assistance in conducting this study.

\section{References}

1 Halpenny D, Courtney K, Torreggiani WC. Dynamic four-dimensional 320 section CT and carpal bone injury - a description of a novel technique to diagnose scapholunate instability. Clin Radiol 2012;67(02):185-187

2 Troupis JM, Amis B. Four-dimensional computed tomography and trigger lunate syndrome. J Comput Assist Tomogr 2013;37(04): 639-643

3 Kwong Y, Mel AO, Wheeler G, Troupis JM. Four-dimensional computed tomography (4DCT): A review of the current status and applications. J Med Imaging Radiat Oncol 2015;59(05): 545-554

4 Gieroba TJ, Bain GI, Cundy PJ. Review of the clinical use of fluoroscopy in hand surgery. Hand Surg 2015;20(02):228-236

5 Bain GI, Hunt J, Mehta JA. Operative fluoroscopy in hand and upper limb surgery: first 100 cases. J Hand Surg Am 1997;22B (05):656-658

6 Galley I, Bain GI, McLean JM. Influence of lunate type on scaphoid kinematics. J Hand Surg Am 2007;32(06):842-847

7 Maarse W, Watts AC, Bain GI. Medium-term outcome following intra-articular corticosteroid injection in first CMC joint arthritis using fluoroscopy. Hand Surg 2009;14(2-3):99-104

8 Zhao K, Breighner R, Holmes D III, Leng S, McCollough C, An KN. A technique for quantifying wrist motion using four-dimensional computed tomography: approach and validation. J Biomech Eng 2015;137(07):074501-1-5

9 Watson HK, Ballet FL. The SLAC wrist: scapholunate advanced collapse pattern of degenerative arthritis. J Hand Surg Am 1984;9 (03):358-365

10 Alta TD, Bell SN, Troupis JM, Coghlan JA, Miller D. The new 4dimensional computed tomographic scanner allows dynamic visualization and measurement of normal acromioclavicular joint 
motion in an unloaded and loaded condition. J Comput Assist Tomogr 2012;36(06):749-754

11 Demehri S, Hafezi-Nejad N, Morelli JN, et al. Scapholunate kinematics of asymptomatic wrists in comparison with symptomatic contralateral wrists using four-dimensional CT examinations: initial clinical experience. Skeletal Radiol 2016;45(04):437-446

12 Garcia-Elias M, Alomar Serrallach X, Monill Serra J. Dart-throwing motion in patients with scapholunate instability: a dynamic fourdimensional computed tomography study. J Hand Surg Eur Vol 2014;39(04):346-352

13 Kakar S, Breighner RE, Leng S, et al. The role of dynamic (4D) CT in the detection of scapholunate ligament injury. J Wrist Surg 2016; 5(04):306-310
14 Wassilew GI, Janz V, Heller MO, et al. Real time visualization of femoroacetabular impingement and subluxation using 320-slice computed tomography. J Orthop Res 2013;31(02):275-281

15 Choi YS, Lee YH, Kim S, Cho HW, Song HT, Suh JS. Four-dimensional real-time cine images of wrist joint kinematics using dual source CT with minimal time increment scanning. Yonsei Med J 2013;54(04):1026-1032

16 Leng S, Zhao K, Qu M, An KN, Berger R, McCollough CH. Dynamic $\mathrm{CT}$ technique for assessment of wrist joint instabilities. Med Phys 2011;38(Suppl 1):S50-S56

17 Biswas D, Bible JE, Bohan M, Simpson AK, Whang PG, Grauer JN. Radiation exposure from musculoskeletal computerized tomographic scans. J Bone Joint Surg Am 2009;91(08):1882-1889 